\title{
Universal technologies in ICT
}

\author{
Frode Eika Sandnes $\cdot$ Suzette Keith · \\ Kevin Yun-Maw Cheng
}

Published online: 22 September 2011

(C) Springer-Verlag 2011

This special issue collects the extended and revised versions of the best papers that were presented at the International Conference on Universal Technologies-Unitech 2010 in Oslo, Norway during May 19-20, 2010. The goal of the Unitech conference was to encourage a stronger focus on the underlying technologies that help support achieving the goal of universal accessibility to information and communication technology. Of the 23 papers accepted for presentation at the conference, 6 are included in this issue in addition to one submitted in response to the open call.

In the paper "On the usefulness of off-the-shelf computer peripherals for people with Parkinson's Disease" Miriam Nes Begnum and Kyrre Begnum provide an assessment of the suitability of commercially available third party peripherals for computer users with physical disabilities, where they advocate the benefits of projector based pointing peripherals.

Several countries are working toward electronic elections, and this move has several ramifications that even may affect democracy. The paper "An evaluation of web-based voting usability and accessibility" by Kristin Skeide Fuglerud and Till Halbach Røssvoll documents experiences with several electronic voting prototypes, looking at

F. E. Sandnes $(\bowtie)$

Faculty of Technology, Art and Design, Oslo and Akershus University College of Applied Sciences, Oslo, Norway e-mail: frodes@hio.no

S. Keith

School of Computing Science, Middlesex University, London, UK

K. Y.-M. Cheng

Department of Computer Science and Engineering,

Tatung University, Taipei, Taiwan implications in the context of reduced functioning, and concluding that designers lack understanding of accessibility guidelines or chose to not prioritize these.

Legislation, standards, guidelines and recommendation are important tools for validating whether systems are universally designed and the W3C WCAG2.0 has received a lot of attention. The paper "Validating WCAG versions 1.0 and 2.0 through Usability Testing with Disabled Users”, by Dagfinn Rømen and Dag Svanæs, describes a study which assessed the effectiveness of WCAG1.0 and WCAG2.0 as heuristics for website accessibility. The results show that WCAG2.0 is only capable of identifying half of the accessibility problems and that WCAG2.0 only is marginally better than WCAG1.0.

Practitioners often speak of how technology needs to be designed to ensure that users with reduced functioning are included, sometimes forgetting the positive sides of new technologies and the unintended but new possibilities that new technologies opens up. In the paper "Universal Life: The Use of Virtual Worlds among People with Disabilities" Kel Smith gives an account of how second life can bring the rest of the world closer to users who are often excluded in the physical world due to their reduced functioning.

Two of the papers in this special issue propose concrete technologies that may help achieve universal access. First, Hugo Fernandes, N Conceição, H. Paredes, A. Pereira, P. Araújo and Joao Barroso, in the paper entitled "Providing accessibility to blind people using GIS", propose a strategy based on geographical information system that provides blind pedestrians enhanced navigation support when moving about outdoors. Second, the paper "EyeBlink Detection System for Human-Computer Interaction" by Aleksandra Krolak and Pawel Strumillo describes an implementation and extensive tests of a system that allows input via eye-blinks for users with physical disabilities who 
may not be able to use conventional peripherals such as keyboards and mice or other modalities such as speech recognition engines.

In the last paper of this issue, entitled "Making touchbased kiosks accessible to blind users through simple gestures", Frode Eika Sandnes, Tek Beng Tan, Anders Johansen, Edvin Sulic, Eirik Vesterhus and Eirik Rud Iversen arguments for the use of simple directional gestures for input on self-service terminals and output via visual and speech cues. They give an account of a prototype and user tests with three groups, namely a control group of people without visual impairment, a group of blindfolded people without visual impairment and a group of visually impaired users. The tests show that most the participants managed to use the system and booked a ticket in less than $4 \mathrm{~min}$.

The guest editors are grateful for the valuable help and advice of the editor-in-chief, Professor Constantine Stephanidis, who also helped manage the review of the guest editor's manuscript. We are also grateful to the reviewers who have put in valuable time to provide constructive advice to the authors that has help ensure and raise the quality of the papers. Finally, we are grateful to all the authors who have chosen this special issue as a forum for communicating their work to a wider audience. 